\title{
Sequence Alignment Map
}

National Cancer Institute

\section{Source}

National Cancer Institute. Sequence Alignment Map. NCI Thesaurus. Code C153248.

A tab-delimited, text-based format for storing biological sequences aligned to a reference sequence. A SAM file includes an optional header section and an alignment section. Each alignment line has 11 mandatory fields for essential alignment information, such as mapping position, and a variable number of optional fields. 